\title{
A note on the coupling of the techni-dilaton to the weak bosons
}

\author{
Michio Hashimotd* \\ Chubu University, \\ 1200 Matsumoto-cho, Kasugai-shi, \\ Aichi, 487-8501, JAPAN \\ (Dated: September 19, 2018)
}

\begin{abstract}
In this note, we study the coupling of the techni-dilaton to the weak bosons. We consider two cases: (1) The dilaton directly couples to the weak bosons similarly to the SM. (2) The coupling in question is effectively induced only through the techni-fermion loops. In both cases, we find that the coupling is essentially determined by the mass-squared of the weak bosons over the dilaton decay constant.
\end{abstract}

PACS numbers: 11.15.Tk, 12.60.Nz, 12.60.Rc, 14.80.Ec

One of the most important aim at the Tevatron and at the CERN Large Hadron Collider (LHC) is to discover the Higgs boson. The direct searches of the standard model (SM) Higgs boson at the LEP have set limits on the Higgs mass to be larger than $114.4 \mathrm{GeV}$ [1]. Recently, the mass ranges of the SM Higgs boson from $114 \mathrm{GeV}$ to $600 \mathrm{GeV}$ have been narrowed down to several windows and slits [2 4]. The fourth generation model [5 7] are also constrained [2, 8]. Besides, these results impact on several classes of the top condensate models [9].

A heavy Higgs boson can be a signal of the existence of models beyond the SM (BSM), because non-standard contributions to the $S$ and $T$ parameters 10 are required for consistency with the LEP precision measurements 1 ]. Such a class of the models contains the walking technicolor (WTC) scenario 11 14.

It is believed that in the WTC, there appears a scalar particle, so-called the techni-dilaton (TD), which is the pseudo Nambu-Goldstone (NG) boson associated with the scale symmetry breaking [12, 15]. The TD mass near the critical point has been suggested as $M_{\mathrm{TD}} \sim \sqrt{2} m$ in the context of the gauged Nambu-Jona-Lasinio (NJL) model [16], where $m$ represents the dynamically generated fermion mass. The TD mass in the criticality limit is discussed recently in Refs. [17, 18].

In the previous work [19], we have studied the yukawa couplings of the SM fermions in the WTC, because the gluon fusion process, which is important in the heavy Higgs searches, depends on the magnitude of the yukawa coupling in addition to the trivial factor arising from the number of the extra heavy colored particles.

In this note, we briefly analyze the coupling of the TD to the weak bosons.

Let us first consider the case that the dilaton $\sigma$ directly couples to $W$. This situation is similar to the SM.

*Electronic address: michioh@isc.chubu.ac.jp

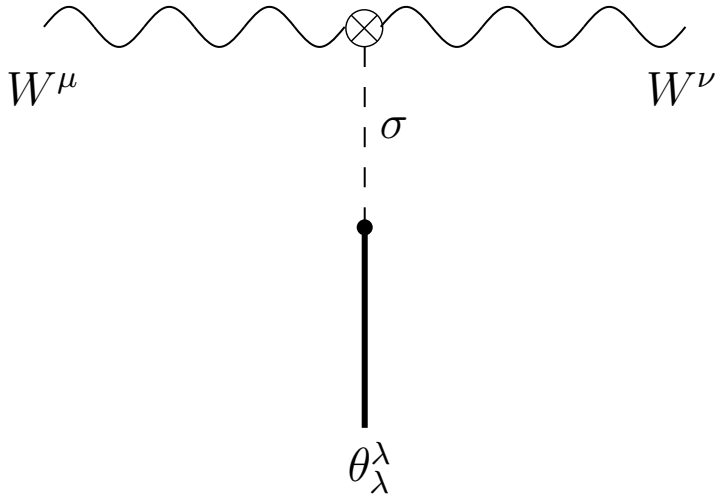

FIG. 1: The $\sigma W W$ coupling in the case that the $\sigma$ directly couples to $W$.

Owing to the nature of the energy-momentum tensor, we formally obtain the following relation [20],

$$
\left\langle W(p)\left|\theta_{\lambda}^{\lambda}(0)\right| W(p)\right\rangle=2 M_{W}^{2} .
$$

Assuming the $\sigma$ dominance at the zero momentum transfer as shown in Fig. 11 we can read the $g^{\mu \nu}$-part of the $\sigma-W^{\mu}-W^{\nu}$ form factor $\Gamma_{\sigma W W}^{\mu \nu}$ as

$$
g_{\sigma W W}(0)=\frac{2 M_{W}^{2}}{F_{\sigma}},
$$

where $F_{\sigma}$ represents the dilaton decay constant being $\left\langle 0\left|\theta_{\lambda}^{\lambda}(0)\right| \sigma(q)\right\rangle=F_{\sigma} M_{\sigma}^{2}$ with the dilaton mass $M_{\sigma}$. The expression (2) agrees with the result in Refs. 21, 22]. For a generalization of Refs. [21, 22], see also Ref. 23].

Next, we study, so-called, the techni-dilaton $\sigma_{T}$ which couples to $W$ only through the techni-fermions (TF's). The axial current $J_{A}^{\mu}$ of the TF's yields the decay constant $F_{\pi},\left\langle 0\left|J_{A}^{\mu}(0)\right| \pi(q)\right\rangle=-i q^{\mu} F_{\pi}$, and the weak boson mass is provided by $F_{\pi}$. We thus consider the coupling between $\sigma_{T}$ and $J_{A}^{\mu}$. 


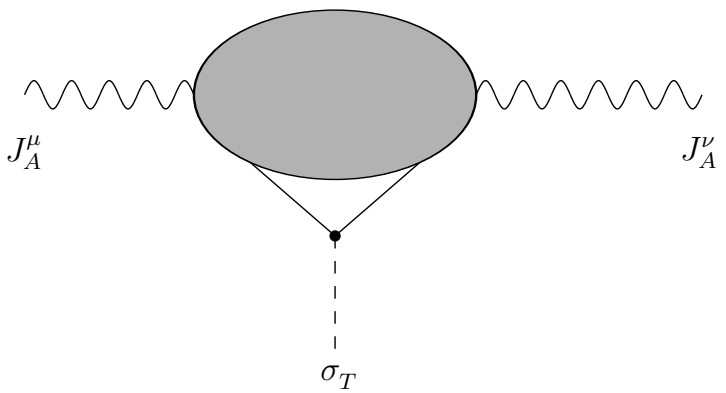

FIG. 2: Coupling of the TD to the axial currents of the TF's. The TD $\sigma_{T}$ couples to $J_{A}^{\mu}$ only through the internal TF lines.

The axial current correlator in the momentum space is

$$
\text { F.T. } i\left\langle 0\left|J_{A}^{\mu}(x) J_{A}^{\nu}(0)\right| 0\right\rangle=\left(g^{\mu \nu}-\frac{q^{\mu} q^{\nu}}{q^{2}}\right) \Pi_{A}\left(q^{2}\right) \text {. }
$$

The vacuum polarization function $\Pi_{A}$ is characterized by

$$
\Pi_{A}(0)=F_{\pi}^{2} .
$$

This relation plays an important role in our approach.

The $\sigma_{T}$ coupling to $J_{A}^{\mu}$ at the zero momentum transfer is just like the mass insertion: Note that the identity holds

$$
\frac{1}{\ell-m} y_{T} \frac{1}{\ell-m}=y_{T} \frac{\partial}{\partial m} \frac{1}{\ell-m}
$$

where $m$ and $y_{T}$ are the dynamically generated TF mass and the yukawa coupling, respectively. We can then obtain the coupling of $\sigma_{T}$ to $J_{A}^{\mu}$ at zero momentum simply by

$$
g_{\sigma_{T} A A}(0)=y_{T} \frac{\partial \Pi_{A}(0)}{\partial m} .
$$

Because $F_{\pi}$ is generated through the TF loop effects, $F_{\pi}$ should be proportional to $m$, i.e., $F_{\pi}=\kappa m$, when we take the infinite limit of the ETC scale. Even in a realistic situation with a finite ETC scale $\sim \mathcal{O}(1000 \mathrm{TeV})$, we expect that $F_{\pi}$ does not strongly depend on the ETC scale. One could find the numerical factor $\kappa$ in Ref. [19], $\kappa \equiv \kappa_{F} \sqrt{N_{\mathrm{TC}}} /(2 \pi)$ with $\kappa_{F} \simeq 1.4-1.5$ and $N_{\mathrm{TC}}$ being the number of the color of the TC gauge group, where the Pagels-Stokar formula [24] is employed. Then Eq. (6) yields

$$
g_{\sigma_{T} A A}(0)=y_{T} \frac{2 F_{\pi}^{2}}{m}
$$

Attaching $W^{\mu}$ to $J_{A}^{\mu}$, we finally obtain the coupling of the TD to the weak bosons at zero momentum,

$$
g_{\sigma_{T} W W}(0)=y_{T} \frac{2 M_{W}^{2}}{m} .
$$

The two cases are conceptually different. However, when the yukawa coupling is like the SM, $y_{T}=m / F_{\sigma}$, Eq. (8) formally agrees with Eq. (2). The yukawa coupling was also estimated as $y_{T}=\left(3-\gamma_{m}\right) m / F_{\sigma}$ with the anomalous dimension $\gamma_{m}(\simeq 1)$ for the model in Ref. [15], where the four-fermion interactions were incorporated, $\mathcal{L}=\mathcal{L}_{\mathrm{TC}}+G_{1}(\bar{T} T)^{2}+G_{2}(\bar{T} T)(\bar{f} f)+G_{3}(\bar{f} f)^{2}$ with $\mathcal{L}_{\mathrm{TC}}$ standing for the TC gauge theory, and $T$ and $f$ being the TF's and the SM fermions, respectively. If so, this suggests that $g_{\sigma_{T} W W}$ is changed by the additional factor $\left(3-\gamma_{m}\right)$ from Eq. (2). Therefore we conclude that the coupling of the TD to the weak bosons is essentially determined by the mass-squared of the weak bosons over the TD decay constant.

Although we have estimated the coupling $g_{\sigma_{(T)} W W}$ at zero momentum, one might expect that the on-shell one is not so far from these estimates. Strictly speaking, the TF mass function in the internal line is not a constant $m$. In sufficiently low energy, however, this would not affect the estimate so much.

The results derived in this note mean that the (effectively induced) operator $\frac{\sigma_{T}}{F_{\sigma}} W_{\mu} W^{\mu}$ yields the coupling between the TD and the weak bosons, similarly to the SM. The earlier argument in Ref. 25] contradicted ours, i.e., they argued that the higher dimensional operator $\frac{\sigma_{T}}{F_{\sigma}} W_{\mu \nu} W^{\mu \nu}$ gave the $\sigma_{T}-W-W$ coupling when the TD couples to $W$ only through the TF-loop. In the end they have revised it, following our results [26].

In any case, the Higgs boson might be revealed soon. What exciting data will be supplied at the LHC and the Tevatron?
[1] K. Nakamura et al. [Particle Data Group], J. Phys. G 37, 075021 (2010).

[2] ATLAS Collaboration, ATLAS-CONF-2011-135 (2011).

[3] CMS Collaboration, CMS PAS HIG-11-022 (2011).

[4] The CDF and the D0 Collaborations, the Tevatron
New Phenomena, and the Higgs Working Group, arXiv:1107.5518 [hep-ex].

[5] H. J. He, N. Polonsky and S. f. Su, Phys. Rev. D 64, 053004 (2001) arXiv:hep-ph/0102144; G. D. Kribs, T. Plehn, M. Spannowsky and T. M. P. Tait, Phys. 
Rev. D 76, 075016 (2007) arXiv:0706.3718 [hep-ph]]; M. Hashimoto, Phys. Rev. D 81, 075023 (2010) arXiv:1001.4335 [hep-ph]]; J. Erler and P. Langacker, Phys. Rev. Lett. 105, 031801 (2010) arXiv:1003.3211 [hep-ph]].

[6] For a dynamical model, see, e.g., M. Hashimoto and V. A. Miransky, Phys. Rev. D 80, 013004 (2009) arXiv:0901.4354 [hep-ph]]; Phys. Rev. D 81, 055014 (2010) arXiv:0912.4453 [hep-ph]].

[7] For reviews, see, e.g., P. H. Frampton, P. Q. Hung and M. Sher, Phys. Rept. 330, 263 (2000) arXiv:hep-ph/9903387; B. Holdom, W. S. Hou, T. Hurth, M. L. Mangano, S. Sultansoy and G. Unel, PMC Phys. A 3, 4 (2009) arXiv:0904.4698 [hep-ph]].

[8] CMS Collaboration, CMS PAS HIG-11-011 (2011); CMS PAS EXO-11-051 (2011).

[9] For a comprehensive review, see, e.g., C. T. Hill and E. H. Simmons, Phys. Rept. 381, 235 (2003); Erratum-ibid. 390, 553 (2004) arXiv:hep-ph/0203079; For a dynamical model with extra dimensions, see, e.g., M. Hashimoto, M. Tanabashi and K. Yamawaki, Phys. Rev. D 64, 056003 (2001); ibid D 69, 076004 (2004); V. Gusynin, M. Hashimoto, M. Tanabashi and K. Yamawaki, Phys. Rev. D 65, 116008 (2002) arXiv:hep-ph/0201106]; For a recent analysis of the topHiggs, see, e.g., R. S. Chivukula, B. Coleppa, H. E. Logan, A. Martin and E. H. Simmons, arXiv:1108.4000 [hep-ph].

[10] M. E. Peskin and T. Takeuchi, Phys. Rev. Lett. 65, 964 (1990); B. Holdom and J. Terning, Phys. Lett. B 247, 88 (1990); M. Golden and L. Randall, Nucl. Phys. B 361, 3 (1991).

[11] B. Holdom, Phys. Rev. D 24, 1441 (1981).

[12] K. Yamawaki, M. Bando and K. Matumoto, Phys. Rev. Lett. 56, 1335 (1986); M. Bando, T. Morozumi, H. So and K. Yamawaki, Phys. Rev. Lett. 59, 389 (1987).

[13] T. Akiba and T. Yanagida, Phys. Lett. B 169, 432 (1986).
[14] T. W. Appelquist, D. Karabali and L. C. R. Wijewardhana, Phys. Rev. Lett. 57, 957 (1986); T. Appelquist and L. C. R. Wijewardhana, Phys. Rev. D 36, 568 (1987).

[15] M. Bando, K. Matumoto and K. Yamawaki, Phys. Lett. B 178, 308 (1986).

[16] S. Shuto, M. Tanabashi and K. Yamawaki, in Proc. 1989 Workshop on Dynamical Symmetry Breaking, Dec. 21-23, 1989, Nagoya, eds. T. Muta and K. Yamawaki (Nagoya Univ., Nagoya, 1990) 115-123; M. S. Carena and C. E. M. Wagner, Phys. Lett. B 285, 277 (1992); M. Hashimoto, Phys. Lett. B 441, 389 (1998). arXiv:hep-th/9805035.

[17] M. Hashimoto and K. Yamawaki, Phys. Rev. D 83, 015008 (2011) arXiv:1009.5482 [hep-ph]].

[18] D. D. Dietrich, F. Sannino and K. Tuominen, Phys. Rev. D 72, 055001 (2005) arXiv:hep-ph/0505059; T. Appelquist and Y. Bai, Phys. Rev. D 82, 071701 (2010) arXiv:1006.4375 [hep-ph]]; L. Vecchi, JHEP 1104, 127 (2011) arXiv:1007.4573 [hep-ph]].

[19] M. Hashimoto, Phys. Rev. D 83, 096003 (2011) arXiv:1103.5576 [hep-ph]].

[20] For a textbook, see, e.g., John F. Donoghue, Eugene Golowich and Barry R. Holstein, "Dynamics of the Standard Model", (Cambridge University Press, Cambridge, 1994).

[21] W. D. Goldberger, B. Grinstein and W. Skiba, Phys. Rev. Lett. 100, 111802 (2008) arXiv:0708.1463 [hep-ph]].

[22] J. Fan, W. D. Goldberger, A. Ross and W. Skiba, Phys. Rev. D 79, 035017 (2009) arXiv:0803.2040 [hep-ph]].

[23] L. Vecchi, Phys. Rev. D 82, 076009 (2010) arXiv:1002.1721 [hep-ph]].

[24] H. Pagels and S. Stokar, Phys. Rev. D 20, 2947 (1979).

[25] S. Matsuzaki and K. Yamawaki, arXiv:1109.1742 [hepph], withdrawn by the authors.

[26] S. Matsuzaki and K. Yamawaki, arXiv:1109.5448 [hep$\mathrm{ph}$. 